\title{
Serum concentration of interleukin 6 is related to inflammation and dyslipidemia in patients with psoriasis
}

\author{
Aldona Pietrzak ${ }^{1}$, Paweł Chabros², Ewelina Grywalska³, Daniel Pietrzak ${ }^{4}$, Grzegorz Kandzierski ${ }^{5}$, \\ Bartłomiej Wawrzycki ${ }^{1}$, Jacek Roliński ${ }^{3}$, Krzysztof Gawęda², Dorota Krasowska ${ }^{1}$
}

\begin{abstract}
${ }^{1}$ Department of Dermatology, Venereology and Pediatric Dermatology, Medical University of Lublin, Lublin, Poland ${ }^{2}$ Department of Orthopedics and Traumatology, Medical University of Lublin, Lublin, Poland ${ }^{3}$ Department of Clinical Immunology and Immunotherapy, Medical University of Lublin, Lublin, Poland ${ }^{4}$ First Clinic of Anesthesiology and Intensive Therapy with Clinical Pediatric Department, Medical University of Lublin, Lublin, Poland ${ }^{5}$ Department of Pediatric Orthopedics and Rehabilitation, Medical University of Lublin, Lublin, Poland
\end{abstract}

Adv Dermatol Allergol 2020; XXXVII (1): 41-45

DOI: https://doi.org/10.5114/ada.2018.78028

\begin{abstract}
Introduction: Patients with psoriasis and psoriatic arthritis (PsA) have metabolic disturbances, which may be due to chronic inflammation.

Aim: Because interleukin-6 (IL-6) regulates both metabolic and inflammatory processes, we evaluated IL-6 as a potential marker of inflammation and metabolic disturbances in psoriasis.

Material and methods: This study involved 93 patients with psoriasis, including 31 patients with concurrent PsA. We investigated whether serum markers of lipid metabolism and inflammation, including IL-6, were related to each other and to disease activity.

Results: We found that concurrent PsA was associated with higher serum concentrations of total cholesterol (TC), low-density lipoprotein cholesterol (LDL-C), and IL-6. In patients with psoriasis alone, the IL- 6 serum concentration correlated positively with the concentrations of TC and LDL-C and with erythrocyte sedimentation rates (ESRs). Moreover, IL-6 concentrations tended to correlate positively with the percentage of the body area affected by psoriatic lesions. Among all patients, those with normal blood lipids had lower ESRs and IL- 6 concentrations than patients with abnormal blood lipids. A logistic regression model showed that PsA, Psoriasis Area Severity Index (PASI), and ESR were significant predictors of the serum IL-6 concentration.

Conclusions: Interleukin- 6 may be an indicator of inflammatory activity in psoriasis. Moreover, IL- 6 may be related to lipid abnormalities in patients with this disease.
\end{abstract}

Key words: psoriasis, psoriatic arthritis, serum interleukin 6, lipids.

\section{Introduction}

In psoriasis, chronic inflammation stimulates the proliferation of cells in the skin, which leads to the development of psoriatic plaques. In about one-third of patients with psoriasis, the disease causes also joint inflammation, typically in the finger and toe joints as well as enthesitis [1]. Moreover, chronic inflammation in psoriasis is associated with metabolic disturbances, such as dys lipidemia [2].

Interleukin 6 (IL-6) is a cytokine with both pro- and anti-inflammatory properties, and it is implicated in the growth and differentiation of epidermal and dermal cells $[3,4]$. Thus, it might be important in the pathogenesis of psoriasis. Indeed, in psoriatic lesions, IL-6 is produced by both inflammatory cells, such as monocytes, dendritic cells, macrophages, and Th17 cells, and by endothelial cells, skin fibroblasts, and keratinocytes [5, 6]. Moreover, the epidermis, fibroblasts, suction blister fluid, and keratinocytes in psoriatic lesions have an increased expression of IL-6 and the IL-6 receptor [3, 7]. Interestingly, IL-6 production is induced by external trauma, which might explain the Koebner's response in patients with psoriasis, i.e. the appearance of skin lesions after skin trauma $[3,4]$. Most previous studies have reported increased serum IL-6 concentrations in patients with psoriasis, particularly in those with active disease [3, 6-15]. The concentration of IL-6 in serum might be related to the severity of both psoriatic skin lesions and joint involvement. This may be because IL-6 promotes inflammatory

Address for correspondence: Aldona Pietrzak PhD, Department of Dermatology, Venereology and Pediatric Dermatology, Medical University of Lublin, 13 Radziwiłłowska St, 20-080 Lublin, Poland, phone: +48 607305 501, e-mail: aldonkapietrzak@o2.pl Received: 28.07.2018, accepted: 6.08.2018. 
cell infiltration in the synovium and synovial hyperplasia [8]. Moreover, IL-6 causes bone resorption, via osteoclast stimulation, and cartilage degeneration, due to the stimulation of matrix metalloproteinases in the synovium and cartilage [16-18].

Chronic psoriatic lesions may produce large amounts of IL- 6 and other pro-inflammatory cytokines, which release free fatty acids, cholesterol, and triglycerides from the adipose tissue [19]. Consequently, patients with psoriasis have increased serum concentrations of triglycerides, total cholesterol (TC), low-density lipoprotein cholesterol (LDL-C), and very low-density lipoprotein cholesterol (VLDL-C).

\section{Aim}

The aim of the study was to evaluate high-sensitivity (HS)-IL6 as a potential marker of inflammation in psoriasis and to relate the serum IL- 6 concentration to the severity and form of psoriasis and to selected metabolic markers.

\section{Material and methods}

\section{Participants}

The study was designed and conducted according to the Declaration of Helsinki and its amendments, current GCP (Good Clinical Practice) guidelines and other appropriate local and international standards and guidelines concerning clinical trials. Moreover, the study was approved by the Local Bioethics Committee at the Medical University of Lublin (decision no. KE-0254/283/2014 of 30 October 2014) and written informed consent was sought from all the study subjects.

We enrolled 93 patients with psoriasis, including 31 with psoriatic arthritis (PsA). Patients who received

Table 1. Age and clinical characteristics of patients with psoriasis alone and with concurrent psoriatic arthritis

\begin{tabular}{|c|c|c|c|c|c|}
\hline \multirow[t]{2}{*}{ Parameter } & \multicolumn{2}{|c|}{$\begin{array}{l}\text { Psoriasis alone } \\
\qquad(n=62)\end{array}$} & \multicolumn{2}{|c|}{$\begin{array}{c}\text { Psoriasis + PsA } \\
(n=31)\end{array}$} & \multirow[t]{2}{*}{$P$-value } \\
\hline & Mean & SD & Mean & SD & \\
\hline Age [years] & 40.87 & 11.05 & 40.68 & 7.38 & 0.93 \\
\hline $\mathrm{SBP}[\mathrm{mm} \mathrm{Hg}]$ & 126.34 & 16.57 & 126.71 & 16.39 & 0.58 \\
\hline $\mathrm{DBP}[\mathrm{mm} \mathrm{Hg}]$ & 79.82 & 14.60 & 83.35 & 10.63 & 0.27 \\
\hline $\begin{array}{l}\text { Psoriasis } \\
\text { duration } \\
\text { [years] }\end{array}$ & 9.25 & 10.12 & 16.75 & 13.19 & $<0.01$ \\
\hline $\begin{array}{l}\text { PsA duration } \\
\text { [months] }\end{array}$ & - & - & 10.35 & 12.91 & - \\
\hline PASI & 26.00 & 6.54 & 28.07 & 5.87 & $<0.05$ \\
\hline BSA & 35.83 & 15.59 & 38.64 & 13.95 & 0.24 \\
\hline
\end{tabular}

medications that could change the concentrations of blood lipids ( $\beta$-blockers, hormones, lipid-lowering drugs, retinoids, dithranol) were excluded. The diagnosis of psoriasis was made based on the "Classification Criteria for Psoriatic Arthritis" (CASPAR). We determined the severity of psoriatic skin lesions with the Psoriasis Area Severity Index (PASI). The percentage of the skin affected by psoriatic lesions was estimated according to the rule that the hand represents $1 \%$ of the total body surface area. We recorded whether the patients smoked cigarettes and drank alcohol.

\section{Laboratory studies}

Blood samples were collected after $12 \mathrm{~h}$ to $14 \mathrm{~h}$ of fasting, and serum was obtained by low-speed centrifugation at $4^{\circ} \mathrm{C}$. The concentrations of glucose, TC, highdensity cholesterol (HDL-C), LDL-C, VLDL-C, and triglycerides (TG) were measured in fresh serum samples with standard diagnostic methods used in our hospital laboratory (Cobas Integra 400; commercially available reagents, Roche Diagnostics, Japan). Enzyme-linked immunosorbent assays (ELISA) were used to measure the serum concentration of IL- 6 in frozen serum samples stored at $-70^{\circ} \mathrm{C}$ until used (Human IL-6 High Sensitivity ELISA Kit, Diaclone, Besancon, France). We calculated the LDL : HDL ratio and the Casteli index according to the following formula: TC/HDL-C. Based on standard cholesterol cut-off, we classified patients into those with normal blood lipids (concentrations of TC $<200 \mathrm{mg} / \mathrm{dl}$, LDL-C $<135 \mathrm{mg} / \mathrm{dl}$, TG < $150 \mathrm{mg} / \mathrm{dl}$ : 35 patients with psoriasis; 13 patients with PsA) and abnormal blood lipids (concentrations of TC $\geq 200 \mathrm{mg} / \mathrm{dl}$, LDL-C $\geq 135 \mathrm{mg} / \mathrm{dl}$, TG $\geq 150 \mathrm{mg} / \mathrm{dl}$ : 27 patients with psoriasis; 18 patients with PsA).

\section{Statistical analysis}

We calculated arithmetic means and standard deviations for quantitative data. The Shapiro-Wilk test was used to check whether the distribution of variables was normal. We compared two groups with the Mann-Whitney $U$-test. The Spearman rank correlation coefficient was calculated for pairs of variables. A logistic regression model (GLZ Multiple Regression Model, Probit Model, Best subsets) was built to find predictors of the serum HS-IL6 concentration. Statistica 12 (StatSoft, Tulsa, OK, United States) was used for all calculations. $P<0.05$ was considered statistically significant.

\section{Results}

Compared to patients with psoriasis alone, those with concurrent PSA had significantly higher PASIs and longer disease duration (Table 1). Concurrent PSA was also associated with higher concentrations of TC, LDL-C, and IL-6 and with higher Castelli indices and LDL : HDL ratios (Table 2). 
Table 2. Metabolic and inflammatory variables in the blood of patients with psoriasis alone and with concurrent psoriatic arthritis

\begin{tabular}{|c|c|c|c|c|c|}
\hline \multirow[t]{2}{*}{ Parameter } & \multicolumn{2}{|c|}{ Psoriasis alone $(n=62)$} & \multicolumn{2}{|c|}{ Psoriasis + PsA $(n=31)$} & \multirow[t]{2}{*}{$P$-value } \\
\hline & Mean & SD & Mean & SD & \\
\hline $\mathrm{BMI}\left[\mathrm{kg} / \mathrm{m}^{2}\right]$ & 26.48 & 4.34 & 27.52 & 4.13 & 0.17 \\
\hline Glucose [mg/dl] & 83.55 & 11.06 & 89.97 & 14.77 & 0.08 \\
\hline Total cholesterol [mg/dl] & 193.54 & 39.75 & 214.27 & 40.08 & $<0.05$ \\
\hline HDL-cholesterol [mg/dl] & 48.40 & 12.15 & 45.69 & 11.59 & 0.25 \\
\hline LDL-cholesterol [mg/dl] & 112.92 & 27.93 & 129.56 & 32.92 & $<0.05$ \\
\hline VLDL-cholesterol [mg/dl] & 25.09 & 10.42 & 33.00 & 19.25 & 0.16 \\
\hline Triglycerides [mg/dl] & 127.70 & 52.95 & 157.21 & 98.01 & 0.56 \\
\hline Castelli index & 4.17 & 1.15 & 5.00 & 1.68 & $<0.05$ \\
\hline LDL : HDL ratio & 2.47 & 0.87 & 3.03 & 1.07 & $<0.05$ \\
\hline $\mathrm{ESR}[\mathrm{mm} / \mathrm{h}]$ & 12.24 & 9.75 & 15.68 & 17.31 & 0.57 \\
\hline HS-IL6 [pg/ml] & 4.21 & 4.05 & 8.79 & 8.19 & $<0.01$ \\
\hline
\end{tabular}

$B M I$ - body mass index, $H D L$ - high density lipoprotein, $L D L$ - low density lipoprotein, VLDL - very low-density lipoprotein, ESR - erythrocyte sedimentation rate, HS-IL6 - high-sensitivity interleukin 6, PSA - psoriatic arthritis.

Table 3. Relationship between serum IL-6 and clinical and laboratory parameters

\begin{tabular}{lcccc}
\hline \multirow{2}{*}{ Parameter } & \multicolumn{2}{c}{ Psoriasis alone } & \multicolumn{2}{c}{ Psoriasis + PsA } \\
\cline { 2 - 5 } & RS & $P$-value & RS & $P$-value \\
\hline Total cholesterol $[\mathrm{mg} / \mathrm{dl}]$ & 0.33 & $<0.05$ & 0.11 & 0.5958 \\
\hline HDL-cholesterol $[\mathrm{mg} / \mathrm{dl}]$ & -0.09 & 0.5377 & 0.20 & 0.3391 \\
\hline LDL-cholesterol $[\mathrm{mg} / \mathrm{dl}]$ & 0.41 & $<0.01$ & -0.08 & 0.6816 \\
\hline VLDL-cholesterol $[\mathrm{mg} / \mathrm{dl}]$ & -0.02 & 0.8766 & -0.20 & 0.3335 \\
\hline Triglycerides $[\mathrm{mg} / \mathrm{dl}]$ & 0.10 & 0.5065 & -0.23 & 0.2559 \\
\hline Castelli index & 0.30 & $<0.05$ & 0.03 & 0.8709 \\
\hline LDL : HDL ratio & 0.28 & $<0.05$ & -0.03 & 0.8918 \\
\hline ESR $[\mathrm{mm} / \mathrm{h}]$ & 0.29 & $<0.05$ & 0.41 & $<0.05$ \\
\hline Psoriasis duration & 0.21 & 0.1384 & -0.05 & 0.7963 \\
\hline PASI & 0.22 & 0.1207 & 0.40 & $<0.05$ \\
\hline BSA & 0.26 & 0.0723 & 0.33 & 0.0980 \\
\hline
\end{tabular}

$H D L$ - high density lipoprotein, $L D L$ - low density lipoprotein, VLDL - very lowdensity lipoprotein, ESR - erythrocyte sedimentation rate, PASI - Psoriasis Area Severity Index, BSA - body surface area (\% of body areas affected by psoriatic lesions), RS - Spearman's rho coefficient.

In patients with psoriasis alone, the IL-6 concentration correlated positively with the concentrations of TC and LDL-C, erythrocyte sedimentation rates (ESRS), Castelli indices, and LDL : HDL ratios (Table 3). Among all patients, only a positive correlation between IL- 6 and ESRS was significant. Both in patients with or without PsA, IL-6 concentrations tended to correlate positively with the percentage of the body area affected by psoriatic lesions, but this relationship was not significant.

Compared to patients with normal blood lipids, those with abnormal blood lipids had higher BMIs, ESRs, and HS-IL6 concentrations (Table 4).
Table 4. Inflammation markers and psoriasis severity in patients with psoriasis with or without psoriatic arthritis according to blood lipid status

\begin{tabular}{lccccc}
\hline Parameter & \multicolumn{2}{c}{$\begin{array}{c}\text { Normal } \\
\text { blood lipids } \\
(n=48)\end{array}$} & \multicolumn{2}{c}{$\begin{array}{c}\text { Abnormal } \\
\text { blood lipids } \\
(n=45)\end{array}$} & $P$-value \\
\cline { 2 - 5 } & Mean & SD & Mean & SD & \\
\hline BMI $\left[\mathrm{kg} / \mathrm{m}^{2}\right]$ & 24.62 & 3.73 & 29.17 & 3.54 & $<0.001$ \\
\hline ESR $[\mathrm{mm} / \mathrm{h}]$ & 10.13 & 9.31 & 16.87 & 15.00 & $<0.01$ \\
\hline HS-IL6 $[\mathrm{pg} / \mathrm{ml}]$ & 4.27 & 5.01 & 7.45 & 6.92 & $<0.05$ \\
\hline PASI & 26.40 & 4.82 & 27.00 & 7.74 & 0.53 \\
\hline BSA & 35.26 & 14.82 & 38.37 & 15.28 & 0.10 \\
\hline BMI & & & & &
\end{tabular}

$B M I$ - body mass index, ESR - erythrocyte sedimentation rate, HS-IL-6 - highsensitivity interleukin 6, PASI - Psoriasis Area Severity Index, BSA - body surface area (\% of body areas affected by psoriatic lesions).

A logistic regression model showed that the clinical form of psoriasis (psoriasis alone vs. psoriasis + PSA), PASI, and ESR were significant predictors of the serum HS-IL6 concentration (Table 5).

\section{Discussion}

Our findings show that IL- 6 may be a marker of both inflammation and disease activity in psoriasis. In our study, the serum IL- 6 concentration correlated with the ESR and tended to be greater in patients with greater percentage skin involvement by psoriatic lesions. Moreover, the ESR, PASI, and PsA were significant independent predictors of the IL- 6 serum concentration. Our study shows that IL-6 might be related to dyslipidemia in psoriasis because patients with abnormal blood lipids had higher IL-6 concentrations than patients with normal blood lipids. 
Table 5. Predictors of the serum IL-6 concentration in a logistic regression model in patients with psoriasis

\begin{tabular}{lcc}
\hline Predictor & Wald & $P$-value \\
\hline $\begin{array}{l}\text { Type of psoriasis (psoriasis alone } \\
\text { vs. psoriasis + PsA) }\end{array}$ & 12.02 & $<0.01$ \\
\hline Blood lipids (normal vs. abnormal) & 0.47 & 0.492 \\
\hline Age [years] & 0.07 & 0.791 \\
\hline Psoriasis duration & 0.32 & 0.569 \\
\hline PASI & 7.11 & $<0.01$ \\
\hline BSA & 0.05 & 0.828 \\
\hline ESR (<10 mm/h; > 10 mm/h) & 3.92 & $<0.05$ \\
\hline Cigarette smoking (yes vs. no) & 1.37 & 0.241 \\
\hline Alcohol consumption (yes vs. no) & 0.20 & 0.655 \\
\hline $\begin{array}{l}\text { PsA - psoriatic arthritis, PASI - Psoriasis Area Severity Index, BSA - body sur- } \\
\text { face area (\% of body areas affected by psoriatic lesions), ESR - erythrocyte } \\
\text { sedimentation rate. }\end{array}$ & &
\end{tabular}

In previous studies, serum IL-6 concentrations were increased in patients with psoriasis and correlated with disease activity assessed with the PASI and DAS28-CRP $[15,17]$. Similarly, we found that IL-6 concentrations were greater in patients with concurrent PsA, which is clinically more severe than plaque psoriasis. The increased IL-6 concentrations in patients with concurrent PsA may be due to additional inflammation in the joints. Moreover, IL-6 concentrations correlated with disease activity (PASI score) in patients with concurrent PsA. Thus, serum IL-6 concentrations may be used to assess psoriasis severity and response to treatment [11]. Based on a study among 182 patients with psoriasis who received biological treatments, Muramatsu et al. suggested that serum concentrations of IL-6 might be particularly valuable for monitoring disease activity in patients who receive infliximab or adalimumab [15]. Moreover, the IL-6 signaling pathway is a potential treatment target in psoriasis [20, 21]. However, tocilizumab, a humanized antibody against the IL- 6 receptor, caused psoriasis-like changes in patients with rheumatoid arthritis. In contrast, clazakizumab, another IL-6 blocker, improved psoriatic arthritis without exacerbating skin lesions [22]. Interleukin-6 blockers might be particularly effective in patients with pustular psoriasis, but the use of these drugs requires further clinical trials [3].

Patients with psoriasis have an increased risk of metabolic diseases, including dyslipidemia, which might be due to chronic inflammation. In this study, in patients with psoriasis, the concentrations of TC and LDL-C, Castelli indices, and LDL: HDL ratios correlated positively with serum IL-6 concentrations, which indicates an association between inflammation and dyslipidemia. Moreover, patients with abnormal blood lipids had significantly higher concentrations of IL-6 and higher ESR values compared to patients with normal blood lipids. Thus, IL- 6 might be implicated in the pathogenesis of psoriasis, lipid disorders, and cardiovascular diseases. These effects of IL-6 might be seen in psoriasis particularly because chronic inflammation may promote the pro-atherogenic effects of IL-6 [23]. For example, in 65 patients with psoriasis, Lise et al. found a correlation between the IL- 6 concentration and the intima-media thickness, an indicator of the cardiovascular risk [14]. Similarly, IL-6 is related to the progression of inflammation and atherosclerosis in coronary arteries [24, 25].

Our study had limitations. First, it was carried out in a single center and was relatively small. Second, we did not include a control group, but the main aim of our study was to investigate whether IL-6 was a marker of inflammation and disease activity in patients with psoriasis.

\section{Conclusions}

Our study showed that IL-6 is a potential marker of disease activity in patients with psoriasis and that IL- 6 might be implicated in the development of lipid abnormalities in these patients. Our findings encourage larger, multi-center studies on the place of IL- 6 in psoriasis.

\section{Acknowledgments}

The authors would like to express their gratitude to Renata Gieroba, PhD for making determinations of interleukin 6, Jolanta Chwaluk Chojnacka MSc for lipid tests, and to the other employees of the Laboratory of Independent Public University Hospital No. 1 (SPSK) in Lublin. The authors would also like to thank Proper Medical Writing sp. z o.o. (www.propermedicalwriting.com) for the assistance in writing this manuscript.

The study was conducted in the Department of Dermatology, Venereology and Pediatric Dermatology, Medical University of Lublin, Poland

\section{Conflict of interest}

The authors declare no conflict of interest.

\section{References}

1. Ogdie A, Weiss P. The epidemiology of psoriatic arthritis. Rheum Dis Clin North Am 2015; 41: 545-68.

2. Pietrzak A, Michalak-Stoma A, Chodorowska G, Szepietowski C. Lipid disturbances in psoriasis: an update. Mediators Inflamm 2010, 2010: 1-13.

3. Saggini A, Chimenti S, Chiricozzi A. IL-6 as a druggable target in psoriasis: focus on pustular variants. J Immunol Res 2014; 2014: 964069.

4. Scheller J, Chalaris A, Schmidt-Arras D, Rose-John S. The proand anti-inflammatory properties of the cytokine interleukin-6. Biochim Biophys Acta 2011; 1813: 878-88.

5. Zalewska A, Głowacka E, Wyczółkowska J, et al. Interleukin 6 and 8 levels in plasma and fibroblast cultures in psoriasis. Mediators Inflamm 2006; 2006: 81767.

6. Pietrzak AT, Zalewska A, Chodorowska G, et al. Cytokines and anticytokines in psoriasis. Clin Chim Acta 2008; 394: 7-21. 
7. Goodman WA, Levine AD, Massari JV, et al. IL-6 signaling in psoriasis prevents immune suppression by regulatory T cells. J Immunol 2009; 183: 3170-6.

8. Dowlatshahi EA, van der Voort EA, Arends LR, Nijsten T. Markers of systemic inflammation in psoriasis: a systematic review and meta-analysis. Br J Dermatol 2013; 169: 266-82.

9. Grossman RM, Krueger J, Yourish D, et al. Interleukin 6 is expressed in high levels in psoriatic skin and stimulates proliferation of cultured human keratinocytes. Proc Natl Acad Sci USA 1989; 86: 6367-71.

10. Neuner P, Urbanski A, Trautinger F, et al. Increased IL-6 production by monocytes and keratinocytes in patients with psoriasis. J Invest Dermatol 1991; 97: 27-33.

11. Bai F, Zheng W, Dong Y, et al. Serum levels of adipokines and cytokines in psoriasis patients: a systematic review and meta-analysis. Oncotarget 2017; 9: 1266-78.

12. Elkayam O, Yaron I, Shirazi I, et al. Serum levels of IL-10, IL-6, IL-1ra, and SIL-2R in patients with psoriatic arthritis. Rheumatol Int 2000; 19: 101-5.

13. Arican O, Aral M, Sasmaz S, Ciragil P. Serum levels of TNFalpha, IFN-gamma, IL-6, IL-8, IL-12, IL-17, and IL-18 in patients with active psoriasis and correlation with disease severity. Mediators Inflamm 2005; 2005: 273-9.

14. Lise MLZ, Baptista TSA, Petersen LE, et al. Subclinical atherogenesis in patients with mild psoriasis: a role for IL-6? Rev Assoc Med Bras 2017; 63: 747-52.

15. Muramatsu S, Kubo R, Nishida E, Morita A. Serum interleukin-6 levels in response to biologic treatment in patients with psoriasis. Mod Rheumatol 2017; 27: 137-41.

16. Tanaka T, Kishimoto T. Targeting interleukin-6: all the way to treat autoimmune and inflammatory diseases. Int J Biol Sci 2012; 8: 1227-36.

17. Ogata A, Kumanogoh A, Tanaka T. Pathological role of interleukin-6 in psoriatic arthritis. Arthritis 2012; 2012: 713618.

18. Feng W, Liu H, Luo T, et al. Combination of IL-6 and SIL-6R differentially regulate varying levels of RANKL-induced osteoclastogenesis through NF-kappaB, ERK and JNK signaling pathways. Sci Rep 2017; 7: 41411.

19. Atzeni F, Ventura D, Batticciotto A, et al. Interleukin 6 blockade: tocilizumab in psoriatic arthritis. J Rheumatol Suppl 2012; 89: 97-9.

20. Laurent S, Le Parc JM, Clerici T, et al. Onset of psoriasis following treatment with tocilizumab. Br J Dermatol 2010; 163: 1364-5.

21. Wendling D, Letho-Gyselinck H, Guillot X, Prati C. Psoriasis onset with tocilizumab treatment for rheumatoid arthritis. J Rheumatol 2012; 39: 657.

22. Mease P, Gottlieb A, Berman A, et al. The efficacy and safety of clazakizumab, an anti-interleukin-6 monoclonal antibody, in a phase Ilb study of adults with active psoriatic arthritis. Arthritis Rheumatol 2016; 68: 2163-73.

23. Pal M, Febbraio M, Whitham M. From cytokine to myokine: the emerging role of interleukin- 6 in metabolic regulation. Immunol Cell Biol 2014; 92: 331-9.

24. Kimura A, Naka T, Kishimoto T. IL-6-dependent and -independent pathways in the development of interleukin 17-producing T helper cells. Proc Natl Acad Sci USA 2007; 104: 12099-104.

25. Yu J, Xiao Z, Zhao R, et al. Astilbin emulsion improves guinea pig lesions in a psoriasis-like model by suppressing IL-6 and IL-22 via p38 MAPK. Mol Med Rep 2018; 17: 3789-96. 